\title{
A ROBUST-TRANSPARENT WATERMARKING SCHEME BASED ON PERCEPTUAL MODELING
}

\author{
P. B. Nguyen, A. Beghdadi, M. Luong \\ Laboratoire de Traitement et de Transport de l'Information (L2TI) \\ Paris 13 University - 99 J.B.Clément, 93430 Villetaneuse, France
}

\begin{abstract}
In this paper, we present a generic watermarking framework that employs perceptual modeling to embed a watermark in such a way that it cannot be perceived. Our approach consists in building computational models which take into account the most common properties of the HVS (Human Visual System) that can be exploited for watermarking. We describe two ways a perceptual model can be incorporated into a watermarking system to control the imperceptibility of the watermark: the implicit and the explicit embedding scheme. Both of schemes are designed in the pyramid transform domain proposed by Burt and Adelson. In the implicit embedding, the watermark is only inserted into imperceptible regions defined using a visibility map. In the explicit embedding, the watermark is embedded in all Laplacian coefficients with strength determined by a JND (Just-Noticeable-Distortion) threshold. The obtained results are twofold. Firstly, the explicit embedding performs better than the implicit one. Secondly, watermarking schemes based on HVS modeling have advantages over schemes that do not take perceptual aspect into consideration. These advantages are demonstrated in terms of imperceptibility and robustness via various experiments.
\end{abstract}

Index Terms - Watermarking, Pyramid Transform, Human Visual System, Visibility Map, JND

\section{INTRODUCTION}

In the last decade, watermarking has received an important consideration, as it is known to be an efficient solution for copyright protection. While a myriad of algorithms have been proposed in recent years, a number of problems still remains. The main issue in watermarking is to solve the trade-off between robustness, capacity and imperceptibility (transparency). Unfortunately, these three criteria are conflicting. Indeed, to enhance robustness, we have to increase the watermark's strength and have loss in transparency and vice versa. Designing algorithms for such an optimizing problem is still a challenge for the watermarking community. We believe that one of the most promising solutions to this challenging issue is to take into account HVS's properties in the design of watermarking algorithms. By this way, we can maximize the robustness when placing a constraint on transparency.

For many years, HVS modeling has become an important issue in image and multimedia processing such as image compression, quality assessment, watermarking and so on. In compression, the limitation of HVS can be exploited for removing redundancy component to achieve high compression rates while maintaining image visual quality. In quality assessment, it seems more natural to exploit HVS's characteristics for evaluation procedure since human observers are the final clients and the key elements in the image/video quality evaluation. Therefore, image/video quality metrics should not be purely based on mathematical model but rather on how human beings judge them. Compared with mathematics based metrics, HVS inspired metrics have demonstrated their better performance and consistency with human perception. In watermarking, there are two major approaches for exploiting HVS's characteristics: implicit and explicit methods. The first approach uses some implicit properties of HVS in the design of an adaptive embedding scheme. The second approach covers methods that use HVS's properties in an explicit and more sophisticated manner to determine the visual detection threshold for embedding watermark signal, i.e. the maximum energy that the watermark can reach without affecting its transparency.

In this study, we proposed two embedding schemes corresponding to these two approaches. These schemes are all derived in the framework of the pyramidal decomposition developed by Burt and Adelson [1]. Such decomposition has been shown to be a powerful tool for analyzing image into multi-scale representation. The paper is organized as follows: In section 2, we describe in detail the implicit embedding scheme as well as the visibility map. In section 3, we propose an explicit embedding that uses the pyramidal JND model. Section 4 demonstrates the performance of our proposed framework and also gives a comparison between the proposed schemes and the Digimarc watermarking technique. Discussions and concluding remarks are finally given in Section 5.

Acknowledgement. This work is partially supported by the HD3D - IIO project of the Cap Digital competitiveness cluster. 


\section{WATERMARKING USING IMPLICIT HVS'S PROPERTIES}

This approach uses some implicit properties of the HVS to determine relevant regions for watermark embedding. Intuitively, a watermark can be well hidden in texture regions than in homogenous regions due to limitation of the HVS. Therefore, the watermark strength can be adaptively determined according to the region activity. In this respect, several HVS based models are successfully exploited for watermarking. In [2], Kutter et al. proposed to use isotropic local contrast and a masking model for calculating the watermark weights. In [3], Voloshynovskiy et al. used the so-called NVF (Noise Visibility Function) which adapts the watermark strength to the local variance of image regions. Thus, the phenomenon underlying these two strategies is that the HVS is less sensitive in high activity regions (texture, edge) than in flat regions. In [4], the watermark is simply embedded in the blue channel of a RGB color image to which the human eye is known to be less sensitive. Kundur et al. [5] used the contrast sensitivity function (CSF) for getting the salience of the image. The strength of the watermark is a function of the salience of the region.

In this section, we proposed an implicit embedding scheme which is based on a visibility map specifically designed for the pyramid decomposition [6]. The image is first decomposed into different scales of the Laplacian Pyramid. The watermark, which is a pseudo-random sequence with zero mean and variance one, is then embedded in some levels of the pyramid. The spread spectrum technique is employed for its proved robustness to common attacks. To guarantee the visual quality, the main idea is to detect and embed the watermark in perceptual irrelevant regions. A visibility map is then determined for each Laplacian level by using the Moon and Spencer local contrast [7] and the Just-Noticeable-Contrast (JNC) [8]. This map allows us to divide each Laplacian image into two zones: the visible and the invisible zones where the watermark can be embedded. Finally, the watermark strength is determined in an adaptive way according to the local contrast.

\subsection{Pyramid Transform}

The pyramid decomposition is first proposed by Burt and Adelson for image compression [1]. It consists of decomposing the image into a set of Gaussian and Laplacian sub-images. The Gaussian Pyramid is a set of low-pass filtered images $G_{0}, G_{l}, \ldots, G_{K}$ where $G_{0}$ is the original image and $G_{k+1}$ is a reduced version of $G_{k}$. Each level of the Gaussian Pyramid (GP) is a smoothed and sub-sampled version of its previous level as follows:

$$
G_{k+1}(i, j)=\operatorname{REDUCE}\left[G_{k}(i, j)\right], k=0,1, \ldots, K-1
$$

The REDUCE operator is a low-pass filtering followed by down-sampling by a factor of two in both the vertical and horizontal directions. The corresponding Laplacian Pyramid
(LP) is computed as the difference between two consecutive levels of the GP whereas the coarser level is "expanded" to the size of the finer level:

$$
L_{k}(i, j)=\left\{\begin{array}{cc}
G_{k}(i, j)-\operatorname{EXPAND}\left[G_{k+1}(i, j)\right] & k=0,1, \ldots, K-1 \\
G_{k}(i, j) & k=K
\end{array}\right.
$$

where EXPAND is the reverse operator of REDUCE, i.e. up-sampling by a factor of two, and then interpolation using the same low-pass filter as in REDUCE.

Here, we set $\mathrm{K}=6$ which is sufficient for describing the number of perceptual channels in the visual cortex. As a result, six Gaussian and five Laplacian sub-images are generated. In the spatial frequency representation, the LP is a set of five quasi-bandpass signals with spatial peak frequencies of $f_{0}, f_{0} / 2, f_{0} / 4, f_{0} / 8, f_{0} / 16, f_{0} / 32$ where $f_{0}$ is the spatial peak frequency of the 1 st level. Here, $f_{0}$ is fixed at 32 cycles/degree(cpd) according to predefined viewing conditions.

Using the pyramid decomposition, the input image can be efficiently represented as a coarse-to-fine hierarchy of decreasing scales in both horizontal and vertical dimension. The original image can be reconstructed as follows:

$$
G_{0}(i, j)=\sum_{k=0}^{K} \operatorname{EXPAND}^{k}\left[L_{k}(i, j)\right]
$$

\subsection{Visibility map}

At each level of the Gaussian pyramid, a visibility map is computed. Let $B$ be an arbitrary luminance levels. The contrast at pixel $(i, j)$ inspired from the Moon and Spencer contrast model [7] is given by [8]:

$$
C(i, j)=\frac{\left|B(i, j)-B_{S}(i, j)\right|}{B_{S}(i, j)}
$$

where $B_{S}(i, j)$ is the immediate surround luminance, calculated as the average luminance of the 8 neighbors of the pixel $(i, j)$ in $3 \times 3$ windows:

$$
B_{S}(i, j)=\frac{1}{8} \sum_{k, l=-1, k, l \neq 0}^{1} B(i+k, j+l)
$$

For the visibility contrast threshold, we make use of the JNC defined in [8]:

$$
J N C(i, j)=\left\{\begin{array}{l}
\frac{C_{w}}{B_{S}(i, j)}\left(A+\sqrt{B_{a}(i, j)}\right)^{2}, B_{a}(i, j) \geq B_{S}(i, j) \\
\frac{C_{w}}{B_{S}(i, j)}\left(A+\sqrt{\frac{B_{S}^{2}(i, j)}{B_{a}(i, j)}}\right)^{2}, B_{a}(i, j)<B_{S}(i, j)
\end{array}\right.
$$

where $C_{w}$ is the Weber-Fechner JNC, $A=0.8$ is an experimentally measured contrast and $B_{a}$ is the adaptation luminance, which is given by:

$$
B_{a}(i, j)=0.923 B_{S}(i, j)+0.077 B_{g}(i, j)
$$


with $B_{g}$ is the global luminance of the image $B$. The visibility map $V$ of the image $B$ is defined as:

$$
V(i, j)=\left\{\begin{array}{l}
1, C(i, j) \geq J N C(i, j) \\
0, C(i, j)<J N C(i, j)
\end{array}\right.
$$

In Fig. 1, the Gaussian, the Laplacian pyramid and the corresponding visibility map are shown.

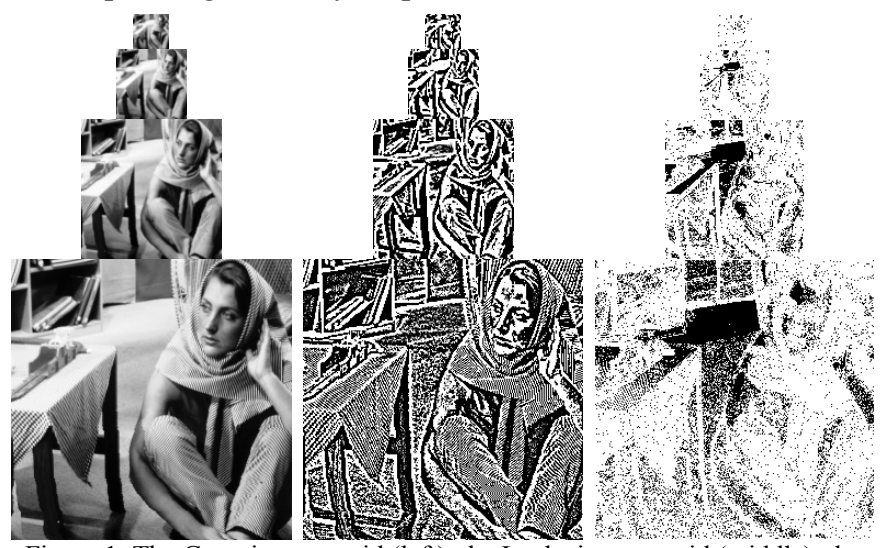

Figure 1. The Gaussian pyramid (left), the Laplacian pyramid (middle), the visibility map pyramid

\subsection{Proposed Adaptive Embedding}

We employ the spread spectrum technique for watermark embedding. The chosen watermark is a pseudo-random sequence which follows a Gaussian distribution of zero mean and variance one. The watermark is inserted into invisible positions $(V(i, j)=0)$ of all levels of the LP using the following expression:

$$
\begin{aligned}
& \hat{L}_{k}(i, j)=L_{k}(i, j)+\alpha \cdot C(i, j) \cdot W(i, j), \\
& \text { if } V_{k}(i, j)=0 \text { with } k=0,1, \ldots, 5
\end{aligned}
$$

Where $V_{k}$ is the visibility map of $G_{k}, C(i, j)$ is the contrast at the $(\mathrm{i}, \mathrm{j})$ position and $\alpha$ is the additional global factor to control the watermark strength. For the sake of security, the watermark is randomly embedded into only $80 \%$ of invisible positions following the secret key. The watermarked image is then constructed as follows:

$$
\hat{G}_{0}=\sum_{k=1}^{K} \operatorname{EXPAND}^{k}\left[\hat{L}_{k}\right]
$$

\section{WATERMARKING USING EXPLICIT HVS'S PROPERTIES}

Implicit embedding employs HVS's characteristics in an indirect manner and most of them require an additional global factor to control the watermark strength. Obviously, the drawback of such an approach is that this global factor is empirically determined and hence varies from an image to another. The second approach covers all of methods that use HVS's properties in an explicit and more sophisticated manner to determine the visual detection threshold for embedding watermark signal. This threshold is generally called JND which refers to the maximum distortion that human eye cannot perceive. In the literature, several JND models have been derived and dedicated to watermarking. In [9], an isotropic local contrast is used in the contrast masking model of Legge and Foley [10] to determine the contrast threshold for each pixel. The JND is then computed as the product of this contrast threshold with the low pass filtered image. In [11], the watermarking scheme proposed by Wolfgang and Podichuk is based on the JND model developed by Watson for DCT coefficients quantization [12].

In this section, we describe a JND model which is also derived for the pyramidal decomposition. Such a model has been successfully used for watermarking [13]. The computed JND threshold is then used to determine an optimal strength for the watermark and hence successfully achieve the trade-off between robustness and imperceptibility.

\subsection{Pyramidal JND Model}

The JND map is computed for each Laplacian level by incorporating the most relevant HVS's properties such as contrast sensitivity, luminance masking and contrast masking.

\subsubsection{Pyramidal Local Band Limited Contrast}

In this section, we propose a new contrast definition based on the local band limited contrast introduced by Peli [14] but adapted for the pyramidal transform. In the pyramidal representation, it is given by:

$$
C_{k}(x, y)=\frac{L_{k}(x, y)}{G_{k+1}(x, y)}
$$

where $L_{k}(x, y)$ and $G_{k}(x, y)$ are the two consecutive Laplacian and Gaussian images, respectively.

\subsubsection{Incorporating Contrast Sensitivity Function (CSF) and Luminance Adaptation}

The CSF describes the variation of HVS's sensitivity as a function of spatial frequency (in cpd) and therefore has to be applied in frequency domain. To adapt this phenomenon for spatial domain, we refer to the approach in [15]. It is known that the early visual stages of HVS work as a multi-channel analyzer and the CSF measured by psychophysical experiments is believed to be the envelope of these channels response [16]. For each channel, the contrast threshold at a given frequency $f$ could be expressed as contrast threshold at the peak frequency $C T\left(f_{k}^{\text {peak }}\right)$ weighted by the contribution of this channel:

$$
C T_{k}(f)=C T_{k}\left(f_{k}^{\text {peak }}\right) \cdot \alpha_{k}(f) \text { where } \alpha_{k}(f)=\frac{L_{k}(f)}{\sum_{k=0}^{K-1} L_{k}(f)}
$$


where $\alpha_{k}(f)$ is the weighting factor and $L_{k}(f)$ is the Laplacian response of the $k^{\text {th }}$ channel. In spatial domain, the local contrast threshold can be approximated as follows:

$$
C T_{k}(x, y)=C T\left(f_{k}^{\text {peak }}\right) \cdot \alpha_{k}(x, y)
$$

where $L_{k}(x, y)$ is the $k^{\text {th }}$ channel's Laplacian response at the pixel $(x, y), C T\left(f_{k}^{\text {paak }}\right)$ is the contrast threshold at the peak frequency of the channel, computed as its inverse CSF.

For computing the CSF, we use the Barten's model [17] thanks to its flexibility and relative simplicity:

$$
\begin{aligned}
& \operatorname{CSF}(f, L)=a \cdot f \cdot \exp (-b . f) \cdot \sqrt{1+c \cdot \exp (b . f)} \\
& \text { where } \quad a=\frac{540(1+0.7 / L)^{-0.2}}{1+\frac{12}{w(1+f / 3)}} \\
& \qquad b=0.3(1+100 / L)^{0.15} \quad c=0.06
\end{aligned}
$$

where $w$ is the angular picture size in degrees and $L$ is the global luminance in $\mathrm{cd} / \mathrm{m} 2$. Previous works treat the contrast sensitivity and the luminance adaptation separately by taking the CSF at a fixed level of illumination followed by a model of luminance masking. However, the CSF exhibits a non-separable dependence between spatial frequency and luminance level. Therefore, we incorporated these two mechanisms into one stage by defining the global luminance $L$ as follows:

$$
L(x, y)=L_{0}+L_{I}(x, y)
$$

where $L_{0}$ is the ambient luminance and $L_{I}$ is the local luminance computed, for each pixel, from the corresponding Gaussian value in the $(k+1)^{\text {th }}$ level $G_{k+1}(x, y)$ followed by a grayscale to luminance transformation as follows:

$$
L_{I}(x, y)=\max \left(L_{\max }\left(\frac{G_{k+1}(x, y)}{255}\right)^{\gamma}, L_{\min }\right)
$$

where $L_{\max }$ and $L_{\min }$ are respectively the maximum and minimum luminance of the display, whereas $\gamma$ is the exponential factor used in the gamma correction of the display.

The computed global luminance, $L(x, y)$, is then putted into equation (14) to account for luminance adaptation.

Finally, based on the contrast definition proposed in Section 3.1.1 the detection threshold $T_{0 k}(x, y)$ which accounts for contrast sensitivity and luminance adaptation is then computed by:

$$
T_{0 k}(x, y)=C T_{k}(x, y) \cdot G_{k+1}(x, y)
$$

\subsubsection{Contrast Masking}

Contrast masking refers to the phenomenon whereby the visibility of a signal is reduced by the presence of another. Here, we propose a contrast masking model inspired from the Legge-Foley's model [10] but we use the new contrast definition for this model:

$$
T_{k}(x, y)=\left\{\begin{array}{l}
T_{0 k}(x, y) \quad i f\left|L_{k}(x, y)\right| \leq T_{0 k}(x, y) \\
T_{0 k}(x, y) \cdot\left(\frac{\left|L_{k}(x, y)\right|}{T_{0 k}(x, y)}\right)^{\varepsilon} \text { otherwise }
\end{array}\right.
$$

where $L_{k}(x, y)$ is the $\mathrm{k}^{\text {th }}$ channel Laplacian response at the pixel $(x, y), \varepsilon$ is a factor that describes the degree of masking, $0.6 \leq \varepsilon \leq 1$ [10]. The final JND threshold is:

$$
J N D_{k}(x, y)=T_{k}(x, y)
$$

For illustration, the JND maps for three Laplacian levels of the "Man" image are shown in Fig. 3.

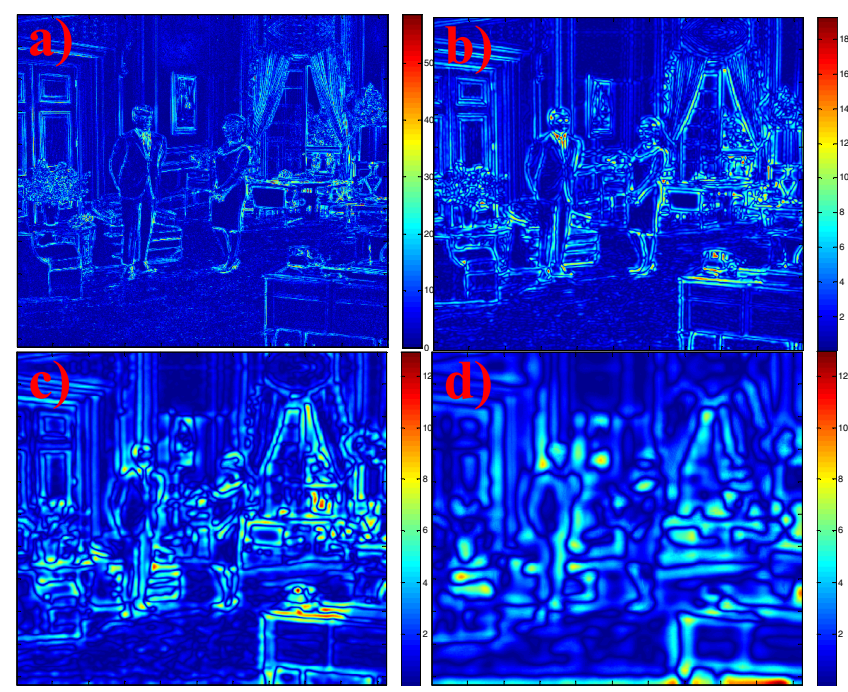

Figure 2. Pyramidal JND map of an image at $32 \mathrm{cpd}$ (a), 16 cpd (b), 8 cpd (c) and 4cpd (d)

\subsection{Explicit Embedding using JND Model}

The embedding scheme is straightforward. The computed JND model in the previous section is used to determine the watermark strength in an additive scheme as below:

At each level, a different bipolar pseudo-random sequence $W_{k} \in\{-1,1\}$ with zero mean and variance one is inserted to the Laplacian image using the rule:

$$
L_{w k}(x, y)=\left\{\begin{array}{l}
L_{k}(x, y)+J N D_{k}(x, y) \cdot W_{k}(x, y) \\
\quad \text { if }\left|L_{k}(x, y)\right|>J N D_{k}(x, y) \\
L_{k}(x, y) \quad \text { otherwise }
\end{array}\right.
$$

The watermarked image is then reconstructed by:

$$
I_{w}=\sum_{k=0}^{K-1} L_{w k}+G^{K}, \quad K=6
$$

For security purpose, the embedding positions are controlled by a pseudo-random key.

\section{DETECTION SCHEME}

Up to now, optimum detectors are only derived under oversimplified assumptions such as in AWGN channels 
where both the host image and the attack noise are modeled as white Gaussian processes [18]. For some other welldefined models like Cauchy, Weibull or Generalized Gaussian distribution (which can fit well to DCT/DWT/Pyramid Transform coefficients), a closed form of the optimum detector can also be derived but only for the absence of attacks. Therefore, due to all difficulties, we opt to use the Linear Correlation Detector (LCD) though it is clearly sub-optimal in our case.

The test image is first decomposed into a set of $K=6$ levels of Laplacian images as in the embedding phase, denoted as $L_{a k}$. The correlation coefficient $\operatorname{Cor}_{k}$ for each level is then defined as:

$$
\operatorname{Cor}_{k}=\frac{\sum_{(x, y)} L_{a k}(x, y) \cdot W_{k}(x, y)}{N_{k}}
$$

where $N_{k}$ is the length of the watermark sequence $W_{k}$. The final correlation coefficient is the maximum correlation value over all levels:

$$
\text { Cor }=\max _{k}\left\{\text { Cor }_{k}\right\}
$$

This correlation value is then compared to a decision threshold $T h$ to decide whether the watermark is present or not. The decision is made according to the hypothesis test below:

$H_{0}$ : the image is not watermarked with $W$

$H_{l}$ : the image is watermarked with $W$.

According to the Neyman-Pearson criterion, this threshold is computed by fixing the false alarm. For simplicity, we consider only the random watermark false alarm. In this case, the detector output follows a Gaussian distribution law. Hence, the false alarm is computed as:

$$
P_{f a}=P\left(\operatorname{Cor}_{s}>T h \mid H_{0}\right)=\frac{1}{2} \operatorname{erfc}\left(\frac{T h}{\sqrt{2} \sigma_{H_{0}}}\right)
$$

$\sigma_{H_{0}}$ is the standard variation of the Laplacian coefficients which is directly estimated on the non-watermarked image using 10000 different watermark sequences. For a false alarm of $10^{-8}$, the decision threshold is $T h=3.9 \sqrt{2} \sigma_{H_{0}}$

\section{PERFORMANCE EVALUATION AND COMPARISON}

In this section, performances of both explicit and implicit schemes are evaluated in terms of transparency and robustness. A comparison with the Digimarc's watermarking technique [19] (which is available as plug-in in the Adobe Photoshop products) is also performed.

\subsection{Transparency Evaluation}

In Fig. 3, the original and the watermarked images from both explicit and implicit schemes as well as Digimarc are shown. We can see that these watermarked images are perceptually undistinguishable from the original one.
Further experiments are also carried out on a set of 8 wellknown images to compare the transparency of the three methods in terms of robustness and imperceptibility.

To evaluate the quality of watermarked images, the DSIS (Double Stimuli Impair Scale) subjective test is performed on 15 observers with the test conditions according to [20]. The grading scale consists of 5 levels as 5: imperceptible, 4: perceptible but not annoying, 3: slightly annoying, 2: annoying, 1: very annoying. Table 1 shows the MOS (Mean Opinion Score) results obtained from three tested methods. It could be noticed that the three watermarking methods achieve a good transparency but the explicit scheme offers the best quality in comparison with other schemes.

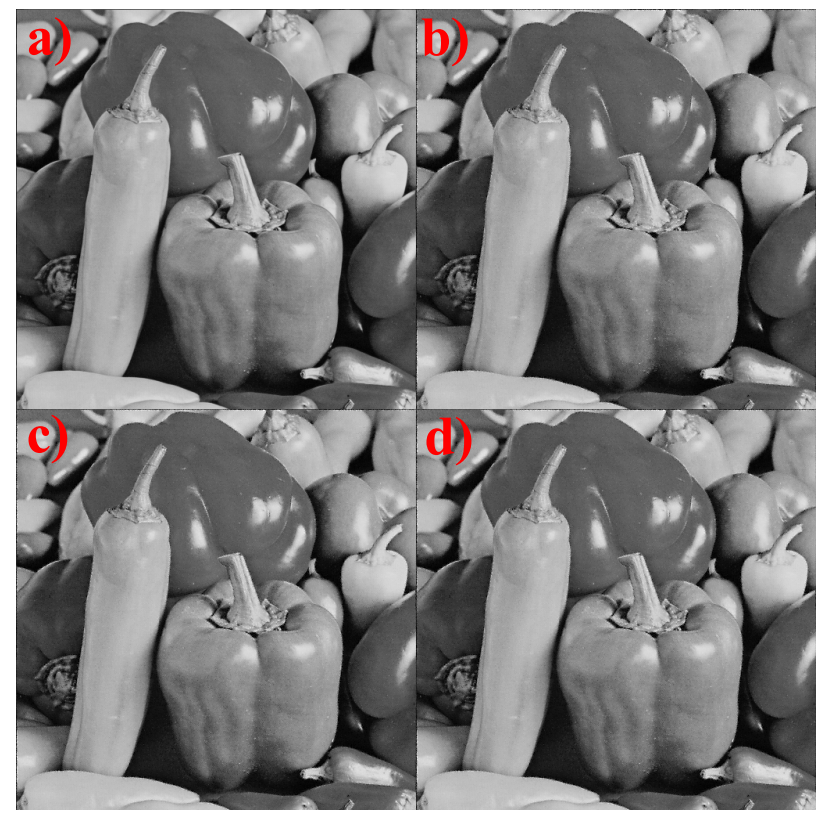

Figure 3. a) Original image and Watermarked images by Explicit scheme (b), Implicit scheme (c) and Digimarc (d)

Table I: Transparency Evaluation by subjective tests

\begin{tabular}{|c|c|c|c|}
\hline Image & Explicit & Implicit & Digimarc \\
\hline Lena & 4.2000 & 3.9333 & 3.6667 \\
\hline Barbara & 4.2000 & 4.0667 & 3.9333 \\
\hline Peppers & 4.8667 & 3.4667 & 3.6000 \\
\hline Parrots & 4.6667 & 4.8667 & 3.6667 \\
\hline Mandrill & 5.0000 & 5.0000 & 3.6000 \\
\hline Man & 4.8667 & 4.2000 & 3.8667 \\
\hline Couple & 4.7333 & 4.4000 & 3.6667 \\
\hline Clown & 4.6667 & 5.0000 & 4.5333 \\
\hline AverageMOS & 4.6500 & 4.3667 & 3.8167 \\
\hline
\end{tabular}

\subsection{Robustness Evaluation}

The robustness of our algorithm is tested via some common attacks from Stirmark [21], Checkmark [22] benchmarks and also some Photoshop manipulations. As shown in Table II, the watermark survived many severe attacks. Throughout these results, it is clear that using perceptual models helps to improve not only transparency but also robustness of a 
watermarking system. The explicit scheme, once again provides the best robustness amongst the compared methods.

Table II: Robustness Evaluation

\begin{tabular}{|c|c|c|c|}
\hline Attack Type & Explicit & Implicit & Digimarc \\
\hline Random Cropping & $0.5 \%$ & $13 \%$ & $34 \%$ \\
\hline Jpeg compression & $\mathrm{QF}=3 \%$ & $\mathrm{QF}=15 \%$ & $\mathrm{QF}=19 \%$ \\
\hline Jpeg 2000 & $0.08 \mathrm{bpp}$ & $0.9 \mathrm{bpp}$ & $0.8 \mathrm{bpp}$ \\
\hline Gaussian Noise & $\sigma=70 \%$ & $\sigma=10 \%$ & $\sigma=4 \%$ \\
\hline Wiener filtering & Passed & Passed & Passed \\
\hline Median filtering & $5 \times 5$ & $3 \times 3$ & Failed \\
\hline Sharpening & Passed & Failed & Failed \\
\hline Blurring & Passed & Failed & Failed \\
\hline Bit plan reduction & Passed & Failed & Failed \\
\hline Histogram Equal. & Passed & Passed & Passed \\
\hline Rescale (50\%) & Passed & Passed & Passed \\
\hline
\end{tabular}

\section{CONCLUDING REMARKS}

We present through this paper two approaches to use HVS models for watermarking: implicit and explicit manner. For the implicit scheme, the main idea consists in embedding of the perceptually invisible watermark by building the map of visibility. A suitable choice of values of the parameters of the watermarking system is proposed to achieve a trade-off between robustness and invisibility. We have also showed that our method can be used to optimize the robustness. However, implicit embedding use the HVS's characteristics in an indirect manner and most of them require an additional global factor to control the watermark strength. Obviously, the drawback of such an approach is that the used global factor is empirically determined and hence varies from an image to another. This drawback is overcome in the explicit embedding scheme by determining the watermark's strength based on a JND threshold. The employed pyramidal JND model is simple and efficient by exploiting principal characteristics of the HVS. The experimental results show that using HVS modeling in watermarking offers a better performance in both transparency and robustness. Especially, using HVS in an explicit manner provides a better result than the implicit embedding scheme.

\section{REFERENCES}

[1] P. J. Burt and E. H. Adelson, "The Laplacian Pyramid as a Compact Image Code", in IEEE Transactions on Communications, April 1983, pp. 532-540.

[2] M. Kutter and S. Winkler, "A Vision-Based Masking Model for Spreadspectrum Image Watermarking,” IEEE Transactions on Image Processing, vol. 11, Issue 1, pp. 16-25, Jan. 2002

[3] S. Voloshynovskiy, A. Herrigel, N. Baumgartner and T. Pun, "A Stochastic Approach to Content Adaptive Digital Image Watermarking", in Proc. of the 3rd International Workshop on Information Hiding, Sept. 1999, pp. 211-236.

[4] M. Kutter, F. Jordan and F. Bossen, "Digital signature of color images using amplitude modulation,” Proc. of SPIEEI97, pp. 518-526, Feb. 1997
[5] D. Kundur and D. Hatzinakos, "A Robust Digital Image Watermarking Method using Wavelet-Based Fusion,” In IEEE-ICIP'97, vol. 1, pp. 544-547, Santa Barbara (Cal), Usa, 1997

[6] Q. B. Do, A. Beghdadi, M. Luong and P. B. Nguyen, “A Perceptual Pyramidal Watermarking Technique", in Proc. of IEEE ICME, Hannover, Germany, June 2008, pp. 281-284.

[7] P. Moon and D. E. Spencer, "The visual effect of non uniform surrounds," J Opt. Soc. Amer. A, vol. 35, pp. 233-248, 1945.

[8] R. Iordache, A. Beghdadi and P.Viaris de Lesengno, "Pyramidal perceptual filtering using moon and spencer contrast," Proceedings of International Conference on Image Processing, vol.3, pp. 146-149, 2001.

[9] M. Kutter and S. Winkler, "A Vision Based Masking Model for SpreadSpectrum Image Watermarking”, in IEEE Transactions on Image Processing, Vol. 11, No. 1, Jan. 2002, pp. 16-25.

[10] G. E. Legge and J. M. Foley, "Contrast Masking in Human Vision”, in Journal of the Optical Society of America 70, 1980, pp. 1458-1471.

[11] R. B. Wolfgang, C. I. Podilchuk, and E. J. Delp, "Perceptual Watermarks for Digital Images and Video," Proceedings of the IEEE, vol. 87, no. 7, Jul. 1999

[12] A. B. Watson, G. Y. Yang, J. A. Solomon, and J. Villasenor, "Visibility of Wavelet Quantization Noise," IEEE Transactions on image processing, vol. 6, no. 8, Aug. 1997.

[13] P. B. Nguyen, A. Beghdadi, and M. Luong, "Perceptual watermarking using pyramidal JND maps", in Proc. of 10th IEEE International Symposium on Multimedia, pp. 418-423, Berkeley, CA, USA (2008).

[14] E. Peli, "Contrast in complex images," Journal of Opt. Soc. Am. A, vol. 7, no. 10, pp. 2032-2040, 1990.

[15] M. Ramasubramanian, S. N. Pattanaik and D. P. Greenberg, "A Perceptually Based Physical Error Metric for Realistic Image Synthesis", in Proc. of SIGGRAPH 99 Conference, Los Angeles, CA, 1999, pp. 73-82.

[16] J. Lubin, "A Visual Discrimination Model for Imaging System Design and Evaluation", in Vision Models for Target Detection and Recognition, E. Peli editor, 1995, pp. 245-283.

[17] P. G. J. Barten, "Evaluation of Subjective Image Quality with the Square-Root Integral Method", in Journal of the Optical Society of America A: Optics, Image Science, and Vision, Vol. 7, Issue 10, Oct. 1990, pp. 2024-2031.

[18] M. Barni and F. Bartolini, "Watermarking Systems Engineering: Enabling Digital Assets Security and Other Applications", CRC Press, ISBN-13: 9780824748067

[19] Digimarc's Watermarking Technologies, https://www.digimarc.com/solutions/dwm.asp

[20] "Methodology for subjective assessment of the quality of television pictures recommendation BT.500-11," International Telecommunication Union, Geneva, Switzerland, 2002.

[21] Stirmark Benchmark, http://www.petitcolas.net/fabien/watermarking/stirmark

[22] Checkmark Benchmark, http://watermarking.unige.ch/Checkmark/index.html 